\title{
Application of microwave radiation in the grafting of acidic sites on SBA-15 type material
}

\author{
Maciej Trejda ${ }^{1}\left[\right.$ ] Magdalena Drobnik ${ }^{1} \cdot$ Ardian Nurwita $^{1}$ (i)
}

Accepted: 2 April 2021 / Published online: 10 April 2021

(c) The Author(s) 2021

\begin{abstract}
Mesoporous silica of SBA-15 type was modified for the first time with 3-(trihydroxysiyl)-1-propanesulfonic acid (TPS) by post-synthesis modification involving microwave or conventional heating in order to generate the Brønsted acidic centers on the material surface. The samples structure and composition were examined by low temperature $\mathrm{N}_{2}$ adsorption/desorption, XRD, HRTEM, elemental and thermal analyses. The surface properties were evaluated by esterification of acetic acid with $\mathrm{n}$-hexanol used as the test reaction. A much higher efficiency of TPS species incorporation was reached with the application of microwave radiation for $1 \mathrm{~h}$ than conventional modification for $24 \mathrm{~h}$. It was found that the structure of mesoporous support was preserved after modification using both methods applied in this study. Materials obtained with the use of microwave radiation showed a superior catalytic activity and high stability.
\end{abstract}

Keywords Ordered silica $\cdot$ TPS $\cdot$ Esterification $\cdot$ Post-synthesis modification

\section{Introduction}

The first syntheses of ordered mesoporous silicas, e.g. MCM-41 [1] or SBA-15 [2] have opened up new research pathways in the field of catalysis. The structure of these solids is characterized by relatively large surface area, e.g. $1000 \mathrm{~m}^{2} \mathrm{~g}^{-1}$, and the presence of hexagonal channels regular in size. The diameter of the channels can be designed by the application of different kind of organic templates that play a role very similar to that of structure directing agent (SDA) in the course of zeolite synthesis. The main areas in which the use of ordered mesoporous materials was considered were heterogenization of different catalytic processes or design the new solid catalysts that could replace earlier used materials [3].

Much attention has been devoted to the development of new catalysts based on silica mesoporous structure and showing acidic properties. In this context, the materials with Brønsted acid sites on their surface are very important and show a variety of possible applications, mainly related to

Maciej Trejda

tmaciej@amu.edu.pl

1 Faculty of Chemistry, Adam Mickiewicz University in Poznań, Uniwersytetu Poznańskiego 8, 61-614 Poznan, Poland the production of the so-called fine chemicals [4]. For this purpose the most often method applied for the synthesis of catalysts having Brønsted acid sites was the immobilization of (3-mercaptopropyl)trimethoxysilane (MPTMS) on the mesoporous silica surface, both using one-pot synthesis method [5, 6] and post-synthesis modification [7, 8]. The advantage of one-pot synthesis modification method is that the oxidation of thiol species takes place in the course of the synthesis of mesoporous material using hydrogen peroxide as an oxidizing agent. It is necessary to generate the acidic $\mathrm{SO}_{3} \mathrm{H}$ species. It was also found that the efficiency of thiol species oxidation can be enhanced for instance by the addition of niobium into the synthesis gel [6]. For post-synthesis modification of ordered mesoporous silica with MPTMS, the oxidation of thiol species has to be performed in a separate step, after MPTMS immobilization. The oxidation process usually involves an excess of hydrogen peroxide. Although the efficiency of thiol species oxidation is usually high a significant part of organosilane modifier can be removed from the silica surface [8]. The mesoporous solids modified with MPTMS are still extensive investigated as catalysts in different processes, e.g. in esterification reactions [9-11] or glycerol dehydration [12], as well as they are applied for adsorption [13] or separation [14] purposes. In this particular work the esterification of acetic acid with $n$-hexanol was applied as a test reaction. Beside the determination of acidity 
of catalysts the product of over-mentioned process, i.e. hexyl acetate, is a valuable product, which can be used for instance as a solvent or paint additive.

In this study the problem with the organosilane removal during oxidation of thiol species after post-synthesis modification was overcome by the application of different kind of organosilane modifier, i.e. 3-(trihydroxysiyl)-1-propanesulfonic acid (TPS), which has already $\mathrm{SO}_{3} \mathrm{H}$ groupings in its structure. To the best of our knowledge this modifier has not been applied for the generation of acidic catalysts using the post-synthesis modification procedure. The goal of this study was not only to obtain an efficient acidic catalyst but also to significantly shorten the time of modification. The post-synthesis modification of mesoporous silica with organosilanes is usually performed within $24 \mathrm{~h}$ by immersing the silica in the mixture of toluene and organosilane [4]. In this study the materials were prepared in a much shorter time, i.e. $1 \mathrm{~h}$, with the application of microwave heating or using the conventional modification procedure.

\section{Materials and methods}

\subsection{Materials}

All chemicals and materials used were purchased from commercially available sources and used without further purification. Tetraethyl orthosilicate-TEOS (>99\%), Pluronic P123, n-hexanol (>99\%), toluene (anhydrous) were purchased from Sigma-Aldrich. 3-(trihydroxysiyl)-1-propanesufonic acid (30-35\% in water) was purchased from Gelest. $\mathrm{HCl}(35 \%)$ and acetic acid were procured from Chempur.

\subsection{Preparation of SBA-15 support}

SBA-15 material was obtained via hydrothermal synthesis. At first a mixture of Pluronic P123 (Poly(ethylene glycol)block-poly(propylene glycol)-block-poly(ethylene glycol) (4 g), $\mathrm{HCl}$ (8.76 g) and water (141.24 g) was prepared. The mixture was kept at $313 \mathrm{~K}$ and then TEOS $(8.527 \mathrm{~g})$ was dropwise added. Finally the mixture was stirred at $313 \mathrm{~K}$ for $20 \mathrm{~h}$ and then heated at $373 \mathrm{~K}$ under static conditions for next $24 \mathrm{~h}$. After synthesis the product was washed with water and dried at RT. The template was removed by calcination at $823 \mathrm{~K}$ for $8 \mathrm{~h}$ (temperature ramp $5 \mathrm{~K} \mathrm{~min}^{-1}$ ).

\subsection{Preparation of the TPS containing catalysts using microwave heating}

Prior to the modification, SBA-15 support was heated at $623 \mathrm{~K}$ for $4 \mathrm{~h}$ in the oven. $1 \mathrm{~g}$ of anhydrous sample was placed in teflon reactor. Next $50 \mathrm{ml}$ of anhydrous toluene and $1.055 \mathrm{~g}$ of TPS solution, i.e. 3-(trihydroxysiyl)-1-propanesufonic acid, were added. The reactor was put into Microwave Labstation (Milestone Microsynth). Mixture obtained was heated at $373 \mathrm{~K}$ (temperature ramp $10 \mathrm{~K} \mathrm{~min}^{-1}$; maximum power $600 \mathrm{~W}$ ) from 1 to $4 \mathrm{~h}$. Next product was separated by filtration and washed subsequently with toluene $(75 \mathrm{ml})$, ethanol $(75 \mathrm{ml})$ and water $(350 \mathrm{ml})$. Finally the product was dried at $373 \mathrm{~K}$ for $12 \mathrm{~h}$.

\subsection{Preparation of the TPS containing catalysts using conventional method}

Prior to the modification, SBA-15 support was heated at $623 \mathrm{~K}$ for $4 \mathrm{~h}$ in the oven. $1 \mathrm{~g}$ of anhydrous sample was placed in the round bottom flask equipped with the reflux. Next $50 \mathrm{ml}$ of anhydrous toluene and $1.055 \mathrm{~g}$ of TPS solution were added. Mixture obtained was heated at $373 \mathrm{~K}$ for $24 \mathrm{~h}$ or $1 \mathrm{~h}$ keeping anhydrous conditions. Next product was separated by filtration and washed subsequently with toluene $(75 \mathrm{ml})$, ethanol $(75 \mathrm{ml})$ and water $(350 \mathrm{ml})$. Finally the product was dried at $373 \mathrm{~K}$ for $12 \mathrm{~h}$.

\subsection{Characterization techniques}

The XRD measurements were performed using a Bruker AXS D8 Advance diffractometer with $\mathrm{Cu} \mathrm{K} \alpha$ radiation $(\lambda=0.154 \mathrm{~nm})$ and at a step of $0.05^{\circ} \mathrm{s}^{-1}$.

For $\mathrm{N}_{2}$ adsorption/desorption measurements the Micromeritics ASAP 2020 instrument was applied. Prior to the $\mathrm{N}_{2}$ adsorption, the sample (ca. $100 \mathrm{mg}$ ) was outgassed at $373 \mathrm{~K}$ under vacuum $(<1.3 \mathrm{~Pa})$ for $20 \mathrm{~h}$. The surface area of samples was calculated using the BET method with 6 points of freedom, the correlation coefficient at least 0.99995 and measurement error less than $2 \mathrm{~m}^{2} \mathrm{~g}^{-1}$. Pore volume and diameter was determined by DFT method.

HR-TEM images were obtained using FEI TecnaiOsiris $200 \mathrm{kV}$ microscope. Prior to the analysis, the powdered samples were suspended in ethanol and deposited on a grid covered with a holey carbon film.

Elemental analyses of the solids were carried out with Elementar Analyser Vario EL III.

Thermogravimetric measurements were carried out in air atmosphere using SETARAM SETSYS-12 apparatus with a temperature ramp of $5 \mathrm{~K} \mathrm{~min}^{-1}$.

\subsection{Esterification processes}

Esterification of acetic acid with n-hexanol was performed in a liquid phase in a glass reactor using an EasyMax Work Station. Prior to the reaction, the catalyst was activated at $423 \mathrm{~K}$ for $12 \mathrm{~h}$ (temperature ramp $5 \mathrm{~K} \mathrm{~min}^{-1}$ ). A mixture of acetic acid (40 mmol) and n-hexanol $(80 \mathrm{mmol})$ was prepared and $100 \mathrm{mg}$ of catalyst was added. The reaction was performed for $4 \mathrm{~h}$ at $373 \mathrm{~K}$. Qualitative analysis was conducted using 
GC-MS chromatograph (Thermo Trace 1300) equipped with DB-1 column $(30 \mathrm{~m})$ to determine possible formation of side products. To determine the product concentration, the residual acid was titrated using $0.1 \mathrm{M}$ solution of $\mathrm{NaOH}$. After the reaction, the catalysts were separated and dried. The most active material was applied in the next catalytic cycle.

\section{Results and discussion}

The aim of this study was to check the possible time reduction of the preparation of acidic catalysts based on SBA-15 type structure modified with organosilanes, in particular with 3-(trihydroxysiyl)-1-propanesulfonic acid (TPS). For this purpose two methodologies were applied. The first one included the application of microwave heating during modification of the support. These samples modified for 1, 2, 3 and $4 \mathrm{~h}$ were labelled as $1 \mathrm{MW}, 2 \mathrm{MW}, 3 \mathrm{MW}$ and $4 \mathrm{MW}$, respectively. The reference sample was prepared in the conventional conditions usually applied according to literature, i.e. with conventional heating for $24 \mathrm{~h}$ (sample denoted as $24 \mathrm{~K}$ ). The second approach was just the time reduction during conventional modification procedure to $1 \mathrm{~h}$, to check the impact of microwave radiation both on efficiency of TPS incorporation and on the stability of SBA-15 structure.

\subsection{Textural/structural characterization}

SBA-15 support was hydrothermally synthesized and shows all characteristic features related to this kind of ordered mesoporous silica. Typical XRD pattern can be observed in Fig. 1 with one intense peak (100) at 2 theta ca. $1^{\circ}$ and two other ones (110) and (200) at 2 theta ca. $1.6^{\circ}$ and $1.9^{\circ}$, respectively. The presence of the first one is related to the reflections of the hexagonal plane group p6mm. It is worth noting that despite the regular size of hexagonal channels, the walls which form the mesoporous silica are amorphous in nature. The presence of next two reflexes of less intensity is a consequence of channels ordering, e.g. their arrangement in the same direction.

It can be observed that the incorporation of TPS using both techniques, i.e. conventional and microwave heating, does not affect the structure of the mesoporous support. All reflexes with similar intensities are still observed in Fig. 1. Only a very slight shift of peaks position towards higher value of 2 theta could be observed after modification with organosilanes. This result is in line with previous data related to the materials obtained with the application of conventional heating [8]. It is well known that the application of microwave heating can cause the so-called "hot spots" in the solution exposed to microwave radiation [15]. It means that locally the temperature during

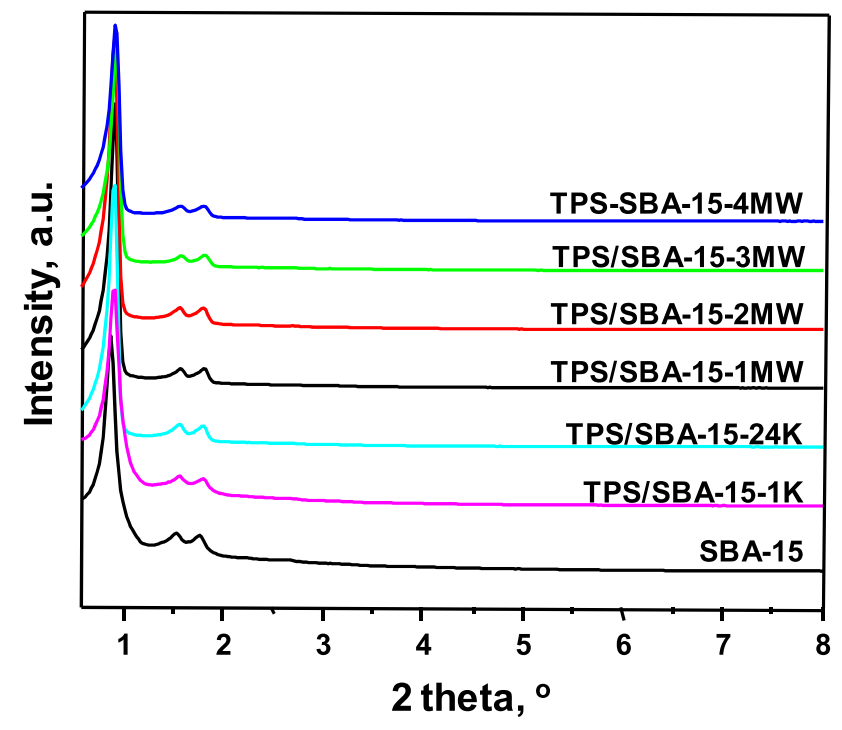

Fig. 1 XRD patterns of materials obtained

the modification can be much higher than the average one measured in the synthesis mixture. One could suppose that this could negatively affect the structure of the support. The results presented in Fig. 1 confirm that the mesoporous structure is untouched after the modification with TPS species.

More information on this matter can be taken from HRTEM measurements. The HR-TEM images of materials obtained are collected in Fig. 2. All samples are found to contain channels regular in size. It can be also seen that these channels are organized forming honeycombs like local structures. These results are in line with the XRD patterns presented in Fig. 1 confirming the preservation of mesoporous structure of silica after modification with TPS regardless the type of technique applied for organosilane incorporation.

The structure and textural parameters of samples obtained were also characterized by low temperature $\mathrm{N}_{2}$ adsorption/ desorption measurements. The isotherms obtained are presented in Fig. 3. The support material shows a typical shape of $\mathrm{N}_{2}$ adsorption/desorption isotherm for SBA-15 materials. It is characterized by the presence of saturation plateau and existence of hysteresis loop. The latter feature is observed due to the condensation of $\mathrm{N}_{2}$ in the material pores. According to IUPAC classification this isotherm can be assigned as a type IVa [16]. The H1 type of hysteresis loop also confirms the presence of a narrow of uniform mesopores, which is in line with XRD and HR-TEM measurements. After modification of SBA-15 with TPS species the same IVa type of $\mathrm{N}_{2}$ adsorption/desorption isotherms is observed. However, the hysteresis loop is of type H5. This distinctive form of hysteresis loop is associated with certain pore structures containing both open and partially blocked mesopores [16]. 

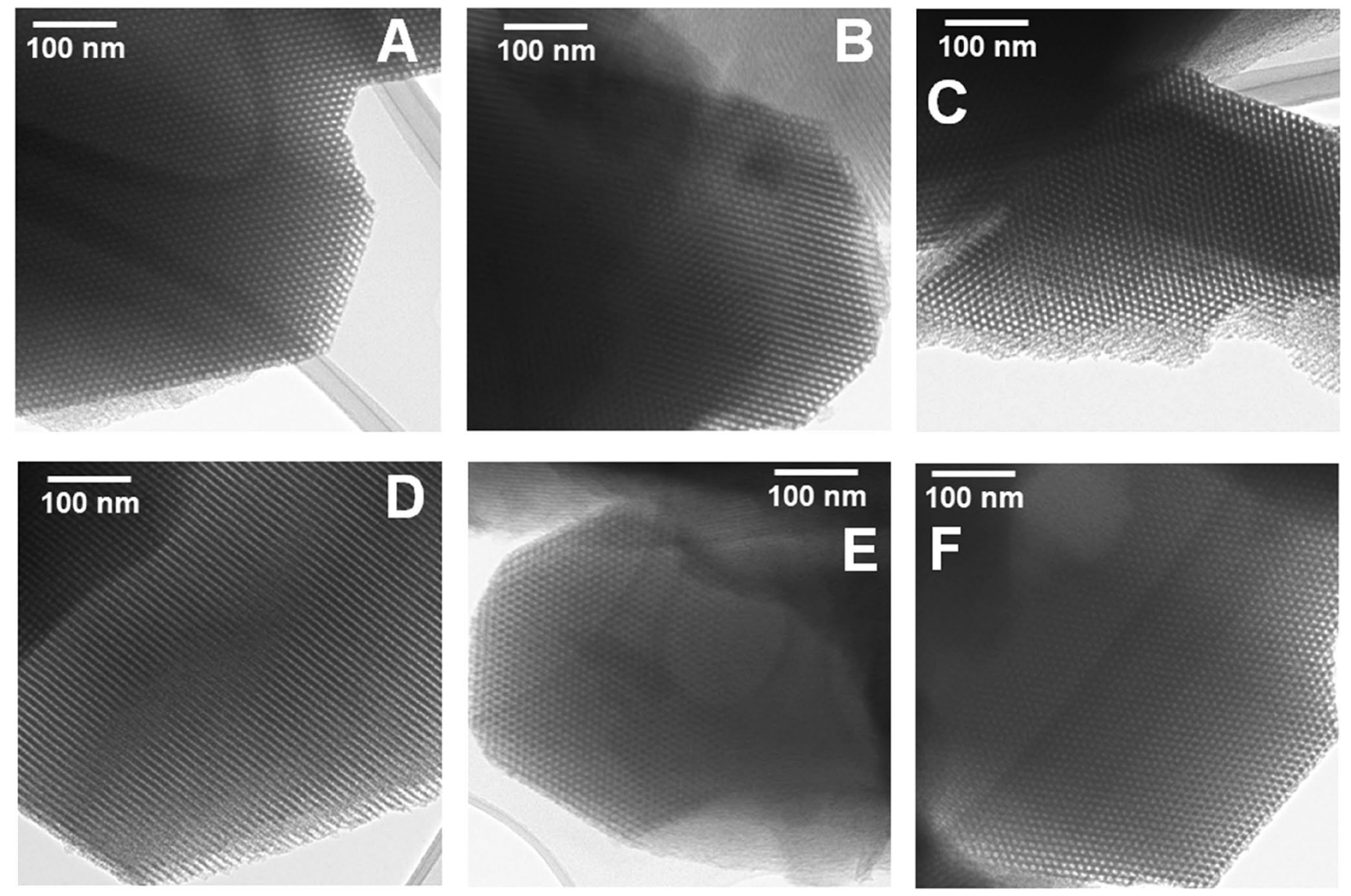

Fig. 2 HR-TEM images of: a TPS/SBA-15-1K; b TPS/SBA-15-24K; c TPS/SBA-15-1MW; d TPS/SBA-15-2MW; e TPS/SBA-15-3MW; f TPS/ SBA-15-4MW
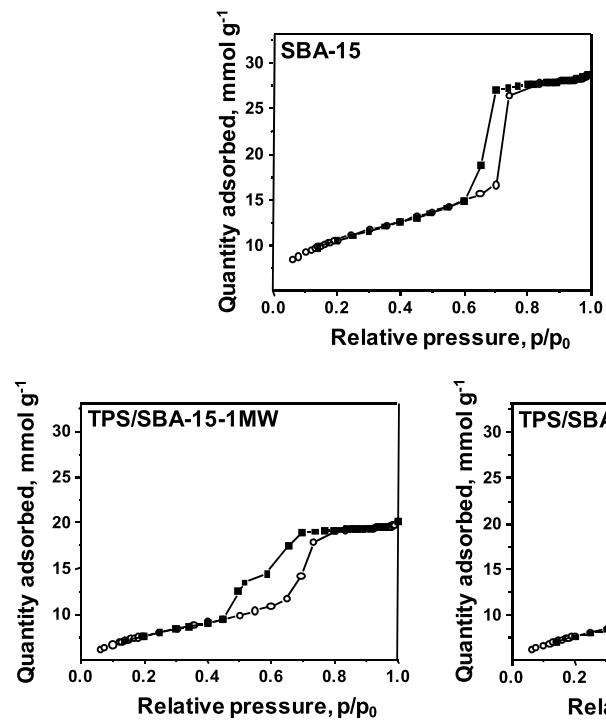
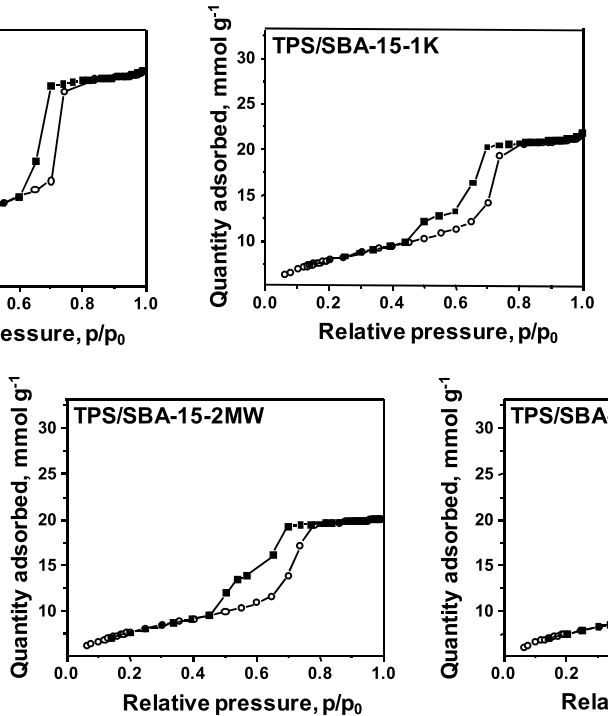
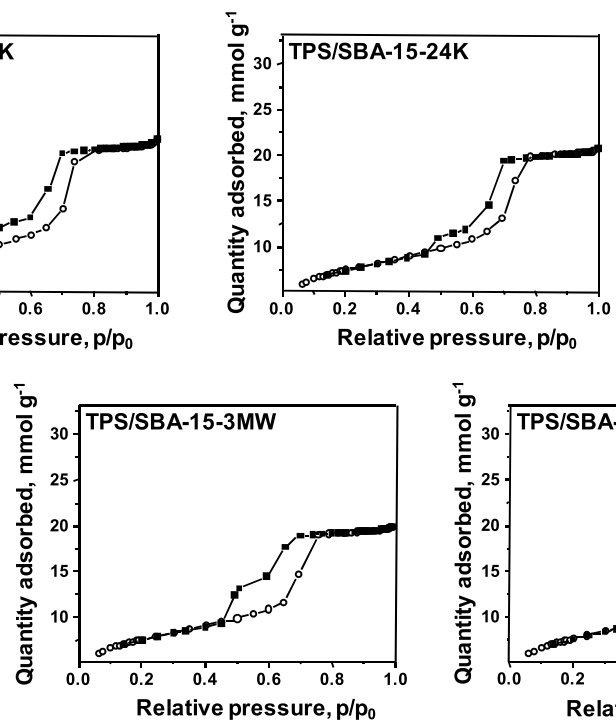

Fig. $3 \mathrm{~N}_{2}$ adsorption/desorption isotherms of SBA-15 and TPS containing materials

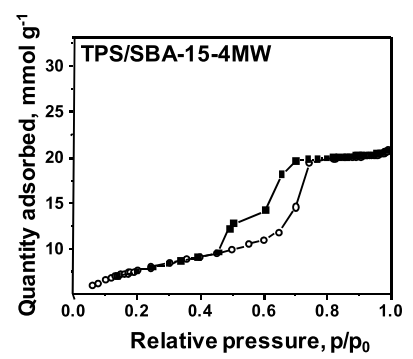


Table 1 Texture/structure characterisation and TPS incorporation efficiency

\begin{tabular}{llllllc}
\hline Catalyst & $\mathrm{S}_{\text {Total }}\left(\mathrm{m}^{2} \mathrm{~g}^{-1}\right)$ & $\mathrm{S}_{\text {micro }}\left(\mathrm{m}^{2} \mathrm{~g}^{-1}\right)$ & $\mathrm{D}(\mathrm{nm})$ & $\mathrm{V}_{\text {Total }}\left(\mathrm{cm}^{3} \mathrm{~g}^{-1}\right)$ & $\mathrm{S}\left(\mathrm{mmol} \mathrm{g}^{-1}\right)$ & Weight loss $(\%)$ \\
\hline SBA-15 & 864 & 199 & 9.7 & 0.96 & - & - \\
TPS/SBA-15-1K & 640 & 154 & 9.6 & 0.71 & 0.61 & 8.1 \\
TPS/SBA-15-24K & 605 & 135 & 9.7 & 0.69 & 0.62 & 8.7 \\
TPS/SBA-15-1MW & 621 & 139 & 9.3 & 0.66 & 0.79 & 10.3 \\
TPS/SBA-15-2MW & 623 & 135 & 9.7 & 0.68 & 0.73 & 10.3 \\
TPS/SBA-15-3MW & 615 & 130 & 9.6 & 0.66 & 0.78 & 10.3 \\
TPS/SBA-15-4MW & 620 & 138 & 9.6 & 0.68 & 0.75 & 10.4 \\
\hline
\end{tabular}

It suggests that in both modification procedures some pore blockage takes place.

The textural parameters calculated from $\mathrm{N}_{2}$ adsorption/ desorption measurements are collected in Table 1. It can be observed that the mesoporous support is characterized by relatively large surface area, i.e. $864 \mathrm{~m}^{2} \mathrm{~g}^{-1}$ and pore volume, i.e. $0.96 \mathrm{~cm}^{3} \mathrm{~g}^{-1}$. The materials after modification with TPS species still show relatively large surface area, however its value decreases by more than $200 \mathrm{~m}^{2} \mathrm{~g}^{-1}$. Irrespectively of the modification time the surface area of the samples prepared with microwave heating is quite similar, ca. $620 \mathrm{~m}^{2} \mathrm{~g}^{-1}$. For the sample obtained by conventional heating (TPS/SBA-15-24 K), some difference in surface area is observed, however one should take into account the difference in synthesis time. The immobilization of TPS species decreases the pore volume, which is in the range between 0.66 and $0.71 \mathrm{~cm}^{3} \mathrm{~g}^{-1}$. This is in line with the type of hysteresis loop observed for these samples, suggesting the existence of some blockage in the pore of mesoporous silica after modification with TPS species.

\subsection{TPS incorporation and acidity of catalysts}

The concentration of TPS species and the number of acidic sites on the SBA-15 support after the modification with the organosilane was estimated by the elemental analysis. The application of this methodology was possible because all sulfur species are present as $\mathrm{SO}_{3} \mathrm{H}$ species when TPS is used for modification (in contrast to MTPMS). Moreover, thermal analysis was conducted to estimate the weight loss related to the removal of the organosilane. The results of both analyses are collected in Table 1. First of all it can be noticed that for all samples the concentration of TPS species is lower than theoretical value based on calculation $\left(1.69 \mathrm{mmol} \mathrm{g}^{-1}\right)$. Moreover, a difference between TPS concentration is observed depending on the method applied for TPS species incorporation. Both analyses clearly indicate that the application of microwave radiation enhances the immobilization of organosilanes species. The concentration of TPS species is in the range between 0.73 and $0.79 \mathrm{mmol}$ of $\mathrm{SO}_{3} \mathrm{H}$ per $1 \mathrm{~g}$ of material. It can be seen that even the shortest modification time allows obtaining a relatively high concentration of TPS species. The method including conventional heating results in concentration of TPS species of ca. 0.61-0.62 mmol of $\mathrm{SO}_{3} \mathrm{H}$ per $1 \mathrm{~g}$ of material. Interestingly, the concentration of TPS species obtained after $1 \mathrm{~h}$ of modification is similar to that obtained after $24 \mathrm{~h}$ that has been usually applied for modification according to literature. It means that the reduction of modification time is possible keeping a very similar efficiency of TPS incorporation. This among others decreases the operation cost. However, it should be emphasize again that much better results are obtained with the application of microwave heating.

\subsection{Acetic acid esterification with $\mathbf{n}$-hexanol}

The acidic properties of materials obtained were tested in liquid phase esterification of acetic acid with n-hexanol. There are two reasons for choosing this reaction. The first is its simplicity as it leads to one main product and therefore makes comparison between catalysts much easier. The possible site products were not detected. The second reason is that both the reaction conditions and chemical nature of

Table 2 Yield of ester in esterification processes of acetic acids with n-hexanol (reaction temp. $373 \mathrm{~K}$; reaction time $4 \mathrm{~h}$; alcohol to acid molar ratio $=2$ )

\begin{tabular}{ll}
\hline Catalyst & $\begin{array}{l}\text { Yield of } \\
\text { hexyl acetate } \\
(\%)\end{array}$ \\
\hline Blank test & 19 \\
TPS/SBA-15-1K & 66 \\
TPS/SBA-15-24K & 68 \\
TPS/SBA-15-1MW & 72 \\
TPS/SBA-15-2MW & 72 \\
TPS/SBA-15-3MW & 73 \\
TPS/SBA-15-4MW & 71 \\
MP/SBA-15* & 52 \\
Nafion SAC 13* & 62 \\
\hline
\end{tabular}

*Results adopted from [17] 


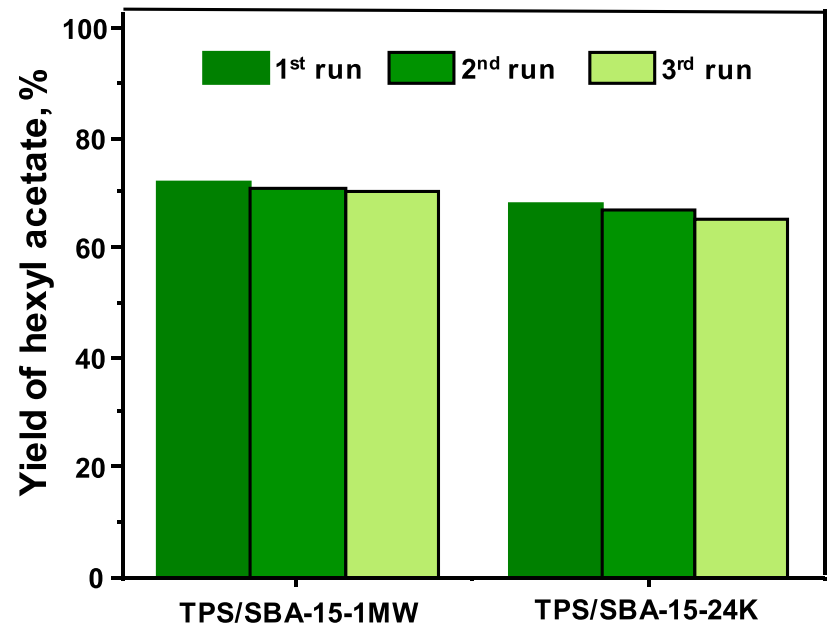

Fig. 4 Stability of TPS/SBA-15-1MW and TPS/SBA-15-24K in three consecutive reactions (reaction temp. $373 \mathrm{~K}$; reaction time $4 \mathrm{~h}$; n-hexanol to acetic acid molar ratio $=2$ )

reactants allow investigation of the stability of organosilane modifier that can be leached from the surface of the support.

The results obtained are presented in Table 2. The esterification process can be driven by the protons coming from the reactants, i.e. in this particular case from the acetic acid. Therefore the reaction performed without the catalysts addition also allows observing the formation of the corresponding ester, however with relatively small yield (19\%). The addition of the solid catalyst significantly improves the conversion of acetic acid, which for most active materials reaches $72-73 \%$. It should be pointed out that the modification of SBA-15 material with TPS species upon microwave radiation for $1 \mathrm{~h}$ allows obtaining a catalyst showing very high activity. This is in line with a higher concentration of acid centers on the surface of the samples obtained with the application of microwave heating than on the surface of those prepared using conventional procedure. Table 2 also presents the results adopted from [17] related to MP/SBA-15 material (obtained from MPTMS) and Nafion SAC 13 often used as a reference sample for liquid phase acid catalyzed processes. It can be noticed that the application of TPS modifier allows obtaining much higher conversion that MPTMS, regardless of the post-synthesis method of catalyst treatment, i.e. microwave or conventional heating. Moreover it can be seen that all the samples show better catalytic performance that Nafion SAC 13.

To check the stability of the modifier on the catalyst surface, two materials, i.e. TPS/SBA-15-24 K and TPS/SBA$15-1 \mathrm{MW}$, after the reaction were separated from the reactant mixture and dried. After reactivation, the catalyst was used in the next catalytic process of acetic acid esterification with n-hexanol. The results obtained are presented in Fig. 4. According to the data very similar yield of hexyl acetate is obtained. This is an important finding because the low stability of active phase on the support can exclude a possible application of such material.

\section{Conclusions}

Two post-synthesis procedures involving microwave or conventional heating applied in this study allowed incorporation of 3-(trihydroxysiyl)-1-propanesulfonic acid (TPS) species on the surface of SBA-15 support. The application of microwave radiation resulted in a significant shortening of modification time with much higher efficiency of TPS incorporation. The characterization of materials proved no negative effect of microwave radiation on the structure of final materials. It was demonstrated that the modification time of mesoporous silica using the conventional procedure can be also shortened keeping almost the same efficiency of incorporation. All materials obtained showed a high activity in esterification of acetic acid with n-hexanol used as a test reaction, much higher than that of Nafion SAC 13. The stability of active phase on the support was also confirmed.

Acknowledgement National Research Center in Poland (Project No. 2017/01/X/ST5/00051) is acknowledged for financial support.

Open Access This article is licensed under a Creative Commons Attribution 4.0 International License, which permits use, sharing, adaptation, distribution and reproduction in any medium or format, as long as you give appropriate credit to the original author(s) and the source, provide a link to the Creative Commons licence, and indicate if changes were made. The images or other third party material in this article are included in the article's Creative Commons licence, unless indicated otherwise in a credit line to the material. If material is not included in the article's Creative Commons licence and your intended use is not permitted by statutory regulation or exceeds the permitted use, you will need to obtain permission directly from the copyright holder. To view a copy of this licence, visit http://creativecommons.org/licenses/by/4.0/.

\section{References}

1. J.S. Beck, J.C. Vartuli, W.J. Roth, M.E. Leonowicz, C.T. Kresge, K.D. Schmitt, C.T.W. Chu, D.H. Olson, E.W. Sheppard, S.B. McCullen, J.B. Higgins, J.L. Schlenker, J. Am. Chem. Soc. 114, 10834 (1992)

2. D. Zhao, Q. Huo, J. Feng, B.F. Chmelka, G.D. Stucky, J. Am. Chem. Soc. 120, 6024 (1998)

3. A. Taguchi, F. Schüth, Micropor. Mesopor. Mater. 77, 1 (2005)

4. P. Gholamzadeh, G. Mohammadi Ziarani, N. Lashgari, A. Badiei, P. Asadiatouei, J. Mol. Catal. A Chem. 391, 208 (2014)

5. D. Margolese, J.A. Melero, S.C. Christiansen, B.F. Chmelka, G.D. Stucky, Chem. Mater. 12, 2448 (2000)

6. M. Trejda, K. Stawicka, M. Ziolek, Appl. Catal. B Environ. 103, $404(2011)$

7. F. Hoffmann, M. Cornelius, J. Morell, M. Fröba, Angew. Chem. Int. Ed. 45, 3216 (2006) 
8. M. Trejda, K. Stawicka, A. Dubinska, M. Ziolek, Catal. Today 187, $129(2012)$

9. B. Lu, Z. Wu, L. Ma, X. Yuan, J. Taiwan Inst. Chem. E. 88, 1 (2018)

10. Y. Noda, K. Li, A.M. Engler, W.A. Elliott, R.M. Rioux, Catal Sci. Technol. 6, 5961 (2016)

11. P. Zhang, H. Wu, M. Fan, W. Sun, P. Jiang, Y. Dong, Fuel 235, 426 (2019)

12. B.O. Dalla Costa, M.S. Legnoverde, C. Lago, H.P. Decolatti, C.A. Querini, Microporous Mesoporous Mater. 230, 66 (2016)

13. S.H. Lee, S.S. Park, S. Parambadath, C.-S. Ha, Micropor. Mesopor. Mater. 226, 179 (2016)

14. A.L. Khan, C. Klaysom, A. Gahlaut, A.U. Khan, I.F.J. Vankelecom, J. Membr. Sci. 447, 73 (2013)
15. A. Loupy, Microwaves in Organic Synthesis (Wiley-VCH Verlag GmbH \& Co KGaA, New York, 2013).

16. M. Thommes, K. Kaneko, A.V. Neimark, J.P. Olivier, F. Rodriguez-Reinoso, J. Rouquerol, K.S.W. Sing, Pure Appl. Chem. 87, 1051 (2015)

17. M. Trejda, A. Nurwita, D. Kryszak, Micropor. Mesopor. Mater. 278, 115 (2019)

Publisher's Note Springer Nature remains neutral with regard to jurisdictional claims in published maps and institutional affiliations. 